\title{
Zygmund spaces, inviscid limit and uniqueness of Euler flows
}

\author{
P.B. MuchA ${ }^{1}$ and W.M. Rusin ${ }^{2}$ \\ 1. Institute of Applied Mathematics and Mechanics, Warsaw University \\ ul. Banacha 2, 02-097 Warszawa, Poland \\ E-mail: p.mucha@mimuw.edu.pl \\ 2. School of Mathematics, University of Minnesota \\ 206 Church Street SE, Minneapolis, 55455 MN, USA \\ E-mail: rusin018@math.umn.edu
}

\begin{abstract}
The paper improves the classical uniqueness result for the Euler system in the $n$ dimensional case assuming that $\nabla u^{E} \in L_{1}(0, T ; B M O(\Omega))$, only. Moreover the rate of the convergence for the inviscid limit of solutions to the Navier-Stokes equations is obtained, provided the same regularity of the limit Eulerian flow. A key element of the proof is a logarithmic inequality between the Hardy and $L_{1}$ spaces which is a consequence of the basic properties of the Zygmund space $\mathbf{L} \ln \mathbf{L}$.
\end{abstract}

\section{Introduction}

The analysis of the evolutionary Euler system modeling the motion of incompressible flows in $n$ dimensional bounded domains is the subject of this paper. We want to study the issue of uniqueness and the problem of the inviscid limit for the Navier-Stokes equations treated as an approximation of the system of inviscid flows.

The classical results [5] and [10] require that solutions to the Euler system should belong at least to the class of regularity which guarantees that the velocity is in the class $u^{E} \in L_{1}\left(0, T ; W_{\infty}^{1}(\Omega)\right)$. Due to that fact we obtain the following estimate

$$
\left|\int_{0}^{T} \int_{\Omega} v \cdot \nabla u^{E} v d x d t\right| \leq C\left\|\nabla u^{E}\right\|_{L_{1}\left(0, T ; L_{\infty}(\Omega)\right)}\|v\|_{L_{\infty}\left(0, T ; L_{2}(\Omega)\right)}^{2}
$$

\footnotetext{
Mathematics Subject Classification (2000). 76B03, 76D09, 35Q30.
}

Keywords. Zygmund spaces, Euler equations, uniqueness, Navier-Stokes equations, inviscid limits. 
which is the core of methods in [5],[10]. Having inequality (1.1) the uniqueness of solutions to the Euler system follows from elementary energy estimates.

The goal of our paper is to improve the classical approach to the Euler system replacing $L_{\infty}$ by the $B M O$ space. Because of relatively low regularity in the studied problem we cannot apply the properties of the $B M O$ space directly. A key element of our technique will be an application of properties of Zygmund spaces $\mathbf{L} \ln \mathbf{L}$ (see [13]). This analysis enables us to prove the following bound

$$
\|w\|_{\mathcal{H}^{1}(\Omega)} \leq C\|w\|_{L_{1}(\Omega)}\left[\left|\ln \|w\|_{L_{1}(\Omega)}\right|+\ln \left(1+\|w\|_{L_{\infty}(\Omega)}\right)\right]
$$

which measures the difference between the $L_{1}$ and Hardy space $\mathcal{H}^{1}$.

We will study the uniqueness criteria which play an important role in analysis based on weak solutions where - by the definition - the high regularity is not admitted. They allow consideration of larger class of external (initial) data to obtain information almost the same as for smooth data. Thanks to (1.2) we will be able to prove that the criteria for the incompressible Euler system should guarantee that $\nabla u^{E} \in L_{1}(0, T ; B M O(\Omega))$, only, replacing the stronger condition from (1.1). Moreover the analysis will enable to consider the approximation of solutions to the Euler system by solutions to the Navier-Stokes equation with small viscosity coefficient. The $B M O$ space is specially distinguished in two dimensions, since it is the limit space for the imbedding $H^{1}\left(\mathbb{R}^{2}\right) \subset B M O\left(\mathbb{R}^{2}\right.$ ) (we can not obtain $L_{\infty}$ here). This case is of our special interest, since we are able to point out good examples for which the $L_{\infty}$ regularity with respect to spatial coordinates is too strong.

Our first result, being the fundamental tool of analysis of the Euler system, is the following

Theorem 1.1 Let $f \in B M O\left(\mathbb{R}^{n}\right)$ and $g \in L_{1}\left(\mathbb{R}^{n}\right) \cap L_{\infty}\left(\mathbb{R}^{n}\right)$, then

$$
\left|\int_{\mathbb{R}^{n}} f g d x\right| \leq C\|f\|_{B M O\left(\mathbb{R}^{n}\right)}\|g\|_{L_{1}\left(\mathbb{R}^{n}\right)}\left[\left|\ln \|g\|_{L_{1}\left(\mathbb{R}^{n}\right)}\right|+\ln \left(1+\|g\|_{L_{\infty}\left(\mathbb{R}^{n}\right)}\right)\right] .
$$

Theorem 1.1 is a version of the logarithmic Sobolev inequality for the Hardy and $L_{1}$ spaces. The structure of (1.3) and its proof is essentially based on the properties of the Zygmund spaces $\mathbf{L} \ln \mathbf{L}$ - see [9],[13]. Inequality (1.2) (or (1.3)) can be compared with a similar estimate between the $L_{\infty}$ and $B M O$ spaces from [6]. The authors have shown that

$$
\|w\|_{L_{\infty}(\Omega)} \leq C\left[1+\|f\|_{B M O(\Omega)}\left(1+\ln ^{+}\|f\|_{W_{p}^{s}(\Omega)}\right)\right] \quad \text { for } \quad s>\frac{n}{p} .
$$

Inequality (1.4) helped to improve the classical result for the Euler system for the blow-up criteria replacing $L_{\infty}$ by the $B M O$ space. In our problems we can not apply estimate (1.4), but it stays the motivation for Theorem 1.1. 
We want to apply Theorem 1.1 to analyze the Euler system

$$
\begin{array}{lr}
u_{t}^{E}+u^{E} \cdot \nabla u^{E}+\nabla p^{E}=0 & \text { in } \Omega \times(0, T), \\
\operatorname{div} u^{E}=0 & \text { in } \Omega \times(0, T), \\
\vec{n} \cdot u^{E}=0 & \text { on } \partial \Omega \times(0, T), \\
\left.u^{E}\right|_{t=0}=u_{0} & \text { in } \Omega .
\end{array}
$$

where $u^{E}: \Omega \times(0, T) \rightarrow \mathbb{R}^{n}$ is the velocity field, $p^{E}: \Omega \times(0, T) \rightarrow \mathbb{R}$ is the pressure, $\vec{n}$ - unit, outward normal to $\partial \Omega, u_{0}: \Omega \rightarrow \mathbb{R}^{n}$ - a divergence-free initial velocity field. We exclude the external forces (the r.h.s. of $(1.5)_{1}$ ), since it would not provide any new analytical difficulties, but only few technical elementary estimates.

We prove the following result concerning the issue of uniqueness of solutions to the system (1.5)

Theorem 1.2 Let $\Omega$ be bounded domain in $\mathbb{R}^{n}$ with smooth boundary. Let $u_{1}^{E}$ and $u_{2}^{E}$ be two solutions to the Euler system (1.5) with initial data $u_{0}$ such that

$$
\begin{gathered}
\nabla u_{1}^{E}, \nabla u_{2}^{E} \in L_{1}(0, T ; B M O(\Omega)) \quad \text { and } \\
u_{1}^{E}, u_{2}^{E} \in L_{\infty}\left(0, T ; L_{2+\sigma}(\Omega)\right) \text { for given } \sigma>0,
\end{gathered}
$$

then $u_{1}^{E} \equiv u_{2}^{E}$.

The above result generalizes the classical theory. The proof of Theorem 1.2 is essentially based on Theorem 1.1 and an application of the Osgood theorem (see [4]) giving uniqueness in the ODE theory. Additionally, the properties of the $B M O$-space allow to exchange the gradient $\nabla u$ by the vorticity rot $u$ in the condition (1.6) which may simplify the application of Theorem 1.1.

An improvement of the classical results as in [5] and [10] can be found [12] and [10]. However the relaxation of the $L_{\infty}$-regularity in the space (in [12] we even have a bit weaker space than $B M O$ ) forces that the regularity with respect to time is required to belong to the $L_{\infty}$-class. So the regularity with respect to time has to be even stronger than in (1.1). Our approach enables to keep the weak condition in the $L_{1}$-norm in $(0, T)$. Moreover thanks to Theorem 1.1 we omit numerous technical estimates which often appear in results of that type.

Our last result concerns the inviscid limit for solutions to the Navier-Stokes equations 
under slip boundary conditions

$$
\begin{array}{lr}
u_{t}^{\nu}+u^{\nu} \cdot \nabla u^{\nu}-\operatorname{div} \mathbb{T}\left(u^{\nu}, p^{\nu}\right)=0 & \text { in } \Omega \times(0, T), \\
\operatorname{div} u^{\nu}=0 & \text { in } \Omega \times(0, T), \\
\vec{n} \cdot \mathbb{T}\left(u^{\nu}, p\right) \cdot \vec{\tau}+\alpha u^{\nu} \cdot \vec{\tau}=0 & \text { on } \partial \Omega \times(0, T), \\
n \cdot u^{\nu}=0 & \text { on } \partial \Omega \times(0, T), \\
\left.u^{\nu}\right|_{t=0}=u_{0} & \text { in } \Omega,
\end{array}
$$

where $u^{\nu}: \Omega \times(0, T) \rightarrow \mathbb{R}^{n}$ is the velocity field, $p^{\nu}: \Omega \times(0, T) \rightarrow \mathbb{R}$ is the pressure, $\vec{n}$ unit, outward normal to $\partial \Omega, \vec{\tau}$ - unit, tangent to $\partial \Omega, \mathbb{T}\left(u^{\nu}, p^{\nu}\right)=\nu \mathbb{D}(u)-p^{\nu}$ Id - stress tensor, $\alpha$-describes friction coefficient of the boundary, $u_{0}: \Omega \rightarrow \mathbb{R}$ - a divergence-free initial velocity field. We are able to consider different boundary conditions than $(1.7)_{3,4}$, however by the results from [1],[7] the form of (1.7) seems to be the most suitable for the issue.

We prove

Theorem 1.3 Let $\Omega$ be bounded domain in $\mathbb{R}^{n}$ with smooth boundary, let $u^{\nu}$ be a solution to the Navier-Stokes system (1.7) and $u^{E}$ be the solution to the Euler system (1.5) both with initial data $u_{0}$. Fix $T>0, \sigma>0$ and consider $\nu$ such that $0<\nu \leq \nu_{0}$. Assume that

$$
\begin{gathered}
\left\|\nabla u^{E}(\cdot, t)\right\|_{B M O(\Omega)} \leq f_{0}(t) \quad \text { and } \quad f_{0} \in L_{1}(0, T), \\
\left\|\nabla u^{\nu}(\cdot, t)\right\|_{L_{2}(\Omega)}+\left\|\nabla u^{E}(\cdot, t)\right\|_{L_{2}(\Omega)} \leq g_{0}(t) \quad \text { and } \quad g_{0} \in L_{2}(0, T), \\
\left\|u^{\nu}(\cdot, t)\right\|_{L_{2+\sigma}(\Omega)}+\left\|u^{E}(\cdot, t)\right\|_{L_{2+\sigma}(\Omega)} \leq h_{0}(t) \quad \text { and } \quad h_{0} \in L_{\infty}(0, T) .
\end{gathered}
$$

Then considering the inviscid limit of solutions to (1.7) we obtain

$$
\sup _{0 \leq t \leq T}\left\|\left(u^{\nu}-u^{E}\right)(\cdot, t)\right\|_{L_{2}(\Omega)} \rightarrow 0 \quad \text { as } \quad \nu \rightarrow 0^{+}
$$

where the precise rate can be expressed by the properties of functions $f_{0}, g_{0}$ and $h_{0}$.

Additionally if we assume extra that

$$
\sup _{0 \leq t \leq T}\left|f_{0}(t)+g_{0}^{2}(t)\right| \leq M
$$

then we obtain the following explicit rate of convergence

$$
\sup _{0 \leq t \leq T}\left\|\left(u^{\nu}-u^{E}\right)(\cdot, t)\right\|_{L_{2}(\Omega)} \leq C \nu^{e^{-2 M T}} .
$$


Theorem 1.3 gives general conditions for the inviscid limit to solutions of the NavierStokes equations, provided very low (lowest known) conditions on the regularity of solutions to (1.7) with respect to the viscous coefficient $\nu$. The main disadvantage is that in the general case we are not able to construct solutions fulfilling (1.8). However in a special case in two dimensions (see [8] and [7] for the case with homogeneous boundary data) we find a class of solutions to (1.7) that fulfills assumptions (1.10). Then by Theorem 1.3 we obtain an explicit rate of convergence to solution of the Euler system given by (1.11). A similar result has been known only for the whole two dimensional case [2] under the classical assumption $\nabla u^{E} \in L_{1}\left(0, T ; L_{\infty}\left(\mathbb{R}^{2}\right)\right)$. The the rate of convergence of $\sup _{0 \leq t \leq T}\left\|u^{\nu}-u^{E}\right\|_{L_{2}\left(\mathbb{R}^{2}\right)}$ is estimated by $\sim \sqrt{\nu T}$, however the initial data considered in [2] correspond to a vortex patch - vorticity is localized to a bounded domain with smooth boundary.

Maybe there is a hope to find a realization of (1.8) by some class of solutions to the Navier-Stokes equations. However the problem seems to be challenging.

In the proceeding, $\Omega$ will be always understood as a bounded subset of $\mathbb{R}^{n}$ with smooth boundary $\partial \Omega$. Spaces $\left(L_{p}(\Omega),\|\cdot\|_{L_{p}(\Omega)}\right),\left(L_{p}\left(\mathbb{R}^{n}\right),\|\cdot\|_{L_{p}\left(\mathbb{R}^{n}\right)}\right)$ for $p \in[1, \infty]$ denote the usual Lebesgue spaces. Spaces $B M O\left(\mathbb{R}^{n}\right)$ and $B M O(\Omega)$ are understood as spaces of measurable functions for which corresponding semi-norms

$$
\|f\|_{B M O\left(\mathbb{R}^{n}\right)}=\sup _{x \in \mathbb{R}^{n}, r>0} f_{B(x, r)}\left|f(s)-\{f\}_{B(x, r)}\right| d s
$$

and

$$
\|f\|_{B M O(\Omega)}=\sup _{x \in \mathbb{R}^{n}, r>0} f_{B(x, r) \cap \Omega}\left|f(s)-\{f\}_{B(x, r) \cap \Omega}\right| d s,
$$

where $\{f\}_{A}=f_{A} f(s) d s=\frac{1}{|A|} \int_{A} d s$ are bounded.

By $C$ we denote a generic constant that is independent from $\nu$.

\section{Proofs of theorems}

We start with the proof of the estimate which plays the key role in next proofs.

Proof of Theorem 1.1. Consider $g \in \mathcal{H}^{1}\left(\mathbb{R}^{n}\right)$. By characterization of $\mathcal{H}^{1}\left(\mathbb{R}^{n}\right)$ we have

$$
\|g\|_{\mathcal{H}^{1}\left(\mathbb{R}^{n}\right)}=\|g\|_{L_{1}\left(\mathbb{R}^{n}\right)}+\sum_{k=1}^{n}\left\|R_{k} g\right\|_{L_{1}\left(\mathbb{R}^{n}\right)},
$$

where the Riesz transform is given in the usual ways as $\mathcal{F}\left[R_{k} f_{k}\right]=\frac{\xi_{k}}{|\xi|} \mathcal{F}\left[f_{k}\right]$. Using the 
fact that $B M O\left(\mathbb{R}^{n}\right)=\left(\mathcal{H}^{1}\left(\mathbb{R}^{n}\right)\right)^{*}$ we get

$$
\left|\int_{\mathbb{R}^{n}} f g d x\right| \leq\|f\|_{B M O\left(\mathbb{R}^{n}\right)}\|g\|_{\mathcal{H}^{1}\left(\mathbb{R}^{n}\right)} \leq\|f\|_{B M O\left(\mathbb{R}^{n}\right)}\left(\|g\|_{L_{1}\left(\mathbb{R}^{n}\right)}+\sum_{k=1}^{n}\left\|R_{k} g\right\|_{L_{1}\left(\mathbb{R}^{n}\right)}\right) .
$$

For the characterization of the Hardy space $\mathcal{H}^{1}\left(\mathbb{R}^{n}\right)$ the reader may refer to [3]. Hence it suffices to obtain an estimate on the $L_{1}$-norm of $R_{k} g$. We use the classical Zygmund result that can be found in [9].

Proposition 2.1 Let $h$ be a sufficiently smooth function with bounded support. Then

$$
\left\|R_{k} h\right\|_{L_{1}\left(\mathbb{R}^{n}\right)} \leq C+C \int_{\mathbb{R}^{n}} h \ln ^{+} h d x
$$

where $\ln ^{+} a=\max \{\ln a, 0\}$ and the constant $C$ depends on the measure of support of $h$.

By elementary scaling we change inequality (2.2) to get

$$
\left\|R_{k} g\right\|_{L_{1}\left(\mathbb{R}^{n}\right)} \leq \lambda+C \int_{\mathbb{R}^{n}} g \ln ^{+} g / \lambda d x
$$

for any $\lambda \in \mathbb{R}_{+}$. Consider $\ln ^{+} g / \lambda=\ln g-\ln \lambda$ for $g \geq \lambda$, then

$$
\left|\ln \left(\left.g\right|_{\{g \geq \lambda\}}\right)\right| \leq\left|\ln \left(1+\|g\|_{L_{\infty}\left(\mathbb{R}^{n}\right)}\right)\right|+\left|\ln \frac{g}{\|g\|_{L_{\infty}\left(\mathbb{R}^{n}\right)}+1}\right|_{\{g \geq \lambda\}} \mid .
$$

Since $\frac{1}{1+\|g\|_{L_{\infty}\left(\mathbb{R}^{n}\right)}} \leq 1$ by elementary properties of logarithms we obtain

$$
\left|\ln \left(1+\|g\|_{L_{\infty}\left(\mathbb{R}^{n}\right)}\right)\right|+\left|\ln \frac{g}{\|g\|_{L_{\infty}\left(\mathbb{R}^{n}\right)}+1}\right|_{\{g \geq \lambda\}}|\leq| \ln \left(1+\|g\|_{L_{\infty}\left(\mathbb{R}^{n}\right)}\right)|+| \ln \frac{\lambda}{\|g\|_{L_{\infty}\left(\mathbb{R}^{n}\right)}+1} \mid .
$$

Since it suffices to consider $\lambda \leq\|g\|_{L_{\infty}\left(\mathbb{R}^{n}\right)}$, we get

$$
\left|\ln \left(\left.g\right|_{\{g \geq \lambda\}}\right)\right| \leq 2 \ln \left(\|g\|_{L_{\infty}\left(\mathbb{R}^{n}\right)}+1\right)+|\ln \lambda| .
$$

Choose $\lambda=\|g\|_{L_{1}\left(\mathbb{R}^{n}\right)}$. We then have

$$
\begin{gathered}
\left\|R_{k} g\right\|_{L_{1}\left(\mathbb{R}^{n}\right)} \leq c\|g\|_{L_{1}\left(\mathbb{R}^{n}\right)}+2 \int_{\mathbb{R}^{n}} g\left(\ln \left(\|g\|_{L_{\infty}\left(\mathbb{R}^{n}\right)}+1\right)+\left|\ln \|g\|_{L_{1}\left(\mathbb{R}^{n}\right)}\right|\right) d x \\
\leq c\|g\|_{L_{1}\left(\mathbb{R}^{n}\right)}\left(1+2 \ln \left(\|g\|_{L_{\infty}\left(\mathbb{R}^{n}\right)}+1\right)+\left|\ln \|g\|_{L_{1}\left(\mathbb{R}^{n}\right)}\right|\right) .
\end{gathered}
$$

Inequality (1.3) follows from inequalities $(2.1),(2.3)$. 
Remark 2.1 Let $\Omega$ be a bounded subset of $\mathbb{R}^{n}, f \in B M O(\Omega), g \in L_{1}(\Omega) \cap L_{\infty}(\Omega)$. Then

$$
\left|\int_{\Omega} f g d x\right| \leq C\|f\|_{B M O(\Omega)}\|g\|_{L_{1}(\Omega)}\left[\left|\ln \|g\|_{L_{1}(\Omega)}\right|+\ln \left(1+\|g\|_{L_{\infty}(\Omega)}\right)\right] .
$$

Proof. Extending $f, g$ by 0 outside $\Omega$ we can apply Theorem 1.1. Now notice that for such extension $\|g\|_{L_{1}\left(\mathbb{R}^{n}\right)}=\|g\|_{L_{1}(\Omega)},\|g\|_{L_{\infty}\left(\mathbb{R}^{n}\right)}=\|g\|_{L_{\infty}(\Omega)}$ and $\|f\|_{B M O\left(\mathbb{R}^{n}\right)} \leq C\|f\|_{B M O(\Omega)}$.

Inequality (1.3) is a key estimate in the proof of Theorem 1.2.

Proof of Theorem 1.2. Let $\left\{u_{1}^{E}, p_{1}^{E}\right\}$ and $\left\{u_{2}^{E}, p_{2}^{E}\right\}$ be two different solutions of (1.5). Subtracting the equations we get

$$
\left(u_{1}^{E}-u_{2}^{E}\right)+u_{1}^{E} \cdot \nabla\left(u_{1}^{E}-u_{2}^{E}\right)+\left(u_{1}^{E}-u_{2}^{E}\right) \nabla u_{2}^{E}=-\nabla\left(p_{1}^{E}-p_{2}^{E}\right) .
$$

Multiplying both sides by $\left(u_{1}^{E}-u_{2}^{E}\right)$ and integrating over $\Omega$ we obtain

$$
\begin{aligned}
& \frac{1}{2} \frac{d}{d t} \int_{\Omega}\left(u_{1}^{E}-u_{2}^{E}\right)^{2} d x+\int_{\Omega}\left(u_{1}^{E}-u_{2}^{E}\right) u_{1}^{E} \nabla\left(u_{1}^{E}-u_{2}^{E}\right) d x \\
& +\int_{\Omega}\left(u_{1}^{E}-u_{2}^{E}\right)^{2} \nabla u_{2}^{E} d x=-\int_{\Omega}\left(u_{1}^{E}-u_{2}^{E}\right) \nabla\left(p_{1}^{E}-p_{2}^{E}\right) d x .
\end{aligned}
$$

Integrating by parts, using boundary conditions and incompressibility of flow we reduce (2.6) to

$$
\frac{1}{2} \frac{d}{d t} \int_{\Omega}\left(u_{1}^{E}-u_{2}^{E}\right)^{2} d x+\int_{\Omega}\left(u_{1}^{E}-u_{2}^{E}\right)^{2} \nabla u_{2}^{E} d x=0 .
$$

Notice that from the assumptions on $u_{1}^{E}, u_{2}^{E}$ and (2.7) it follows that $\left\|u_{1}^{E}-u_{2}^{E}\right\|_{L_{2}(\Omega)} \in$ $C([0, T])$. We split $\alpha=\alpha_{m}+\alpha_{r}$ where $\left|\alpha_{m}\right|=\min (|\alpha|, m)$ for some $m>1$. Notice that we can extend all functions outside $\Omega$ by 0 . Upon Theorem 1.1 and Remark 2.1 we get

$$
\left|\int_{\Omega}\right| \alpha \beta|d x| \leq C\|\beta\|_{B M O(\Omega)}\left\|\alpha_{m}\right\|_{L_{1}(\Omega)}\left(1+\left|\ln \left\|\alpha_{m}\right\|_{L_{1}(\Omega)}\right|+\ln (1+m)\right)+\int_{\Omega}\left|\alpha_{r} \beta\right| d x .
$$

Denote $f(t)=2 C\|\beta(t)\|_{B M O}, g(t)=\int_{\Omega}\left|\alpha_{r} \beta\right| d x, x(t)=\left\|u_{1}^{E}-u_{2}^{E}\right\|_{L_{2}(\Omega)}^{2}$. Consider $x(t)$ small enough so that the function $|x(t) \ln x(t)|$ is increasing (which by the continuity of $x(t)$ is equivalent to restricting our attention to sufficiently small $T$ ), then (2.8) can be restated as follows

$$
\left|\int_{\Omega}\right| \alpha \beta|d x| \leq C\|\beta\|_{B M O(\Omega)}\|\alpha\|_{L_{1}(\Omega)}\left(1+\left|\ln \|\alpha\|_{L_{1}(\Omega)}\right|+\ln (1+m)\right)+\int_{\Omega}\left|\alpha_{r} \beta\right| d x .
$$


Thus from (2.9) we obtain the following inequality

$$
\begin{aligned}
& \dot{x} \leq f(t) x(t)(|\ln x(t)|+1+\ln (1+m))+g(t), \\
& x(0)=0 .
\end{aligned}
$$

To find a good estimate on $x(t)$ we introduce the following equation

$$
\begin{aligned}
& \dot{y}=f(t) y(t)(|\ln y(t)|+1+\ln (1+m))+g(t), \\
& y(0)=1 / m,
\end{aligned}
$$

for some $m$ large enough. From the Osgood existence theorem we know that there exists a unique local solution to (2.11). Additianlly the r.h.s. of $(2.11)_{1}$ guarantees that $y(\cdot)$ is increasing. It implies that the solution of (2.11) majorizes $x(t)$, i.e.:

$$
0 \leq x(t) \leq y(t) \quad \text { for } \quad t \in[0, T]
$$

Hence we investiagate the bahavior of the solutions to (2.11). By Gronwall's inequality we get

$$
\begin{gathered}
y(t) \leq \frac{1}{m} \exp \left(\int_{0}^{t} f(s)(|\ln y(s)|+1+\ln (1+m)) d s\right)+ \\
\int_{0}^{t} g(s) \exp \left(\int_{s}^{t} f(\tau)(|\ln y(\tau)|+1+\ln (1+m)) d \tau\right) d s \leq \\
\frac{1}{m} \exp \left((1+\ln (1+m)) \int_{0}^{t} f(s) d s\right) \exp \left(\int_{0}^{t} f(s)|\ln y(s)| d s\right)+ \\
+\exp \left(\int_{0}^{t} f(\tau)|\ln y(\tau)| d \tau\right) \exp \left((1+\ln (1+m)) \int_{0}^{t} f(\tau) d \tau\right) \int_{0}^{t} g(s) d s .
\end{gathered}
$$

Since $y(t) \geq 1 / m$ implies $|\ln y(t)| \leq \ln m$ we can estimate the right hand-side (modulo some constant) of (2.12) by

$$
\begin{gathered}
\frac{1}{m}(1+m)^{\int_{0}^{t} f(s) d s} m^{\int_{0}^{t} f(s) d s}+m^{\int_{0}^{t} f(s) d s}(1+m)^{\int_{0}^{t} f(s) d s} \int_{0}^{t} g(s) d s= \\
(m(1+m))^{\int_{0}^{t} f(s) d s}\left(\frac{1}{m}+\int_{0}^{t} g(s) d s\right) \leq \\
\left(2 m^{2}\right)^{\int_{0}^{t} f(s) d s}\left(\frac{1}{m}+\int_{0}^{t} \int_{\Omega}\left|\alpha_{r} \beta\right| d x d s\right) .
\end{gathered}
$$

This shows that it suffices to control the part $\alpha_{r}$. In this case we have estimates on the measure of the support. Since $\alpha \in L_{\infty}\left(0, T ; L_{1+\sigma / 2}(\Omega)\right)$ and $\beta \in B M O(\Omega)$ hence $\beta \in L_{p}(\Omega)$ for any $p<\infty$ we have by elementary Hölder's inequality

$$
\int_{\Omega}\left|\alpha_{r} \beta\right| d x \leq\left\|\alpha_{r}\right\|_{L_{1+\sigma / 4}(\Omega)}\|\beta\|_{L_{(1+\sigma / 4)^{\prime}}(\Omega)}
$$


hence we obtain a bound

$$
\begin{gathered}
\left(2 m^{2}\right)^{\int_{0}^{t} f(s) d s}\left(\frac{1}{m}+\int_{0}^{t} \int_{\Omega}\left|\alpha_{r} \beta\right| d x d s\right) \\
\leq\left(2 m^{2}\right)^{\int_{0}^{t} f(s) d s}\left(\frac{1}{m}+\left\|\alpha_{r}\right\|_{L_{\infty}\left(0, T ; L_{1+\sigma / 4}(\Omega)\right)}\|\beta\|_{L_{1}\left(0, T ; L_{(1+\sigma / 4)^{\prime}}(\Omega)\right)}\right) .
\end{gathered}
$$

From Chebyschev inequality we have

$$
\left|\operatorname{supp} \alpha_{r}\right| \leq\left(\frac{\|\alpha\|_{L_{\infty}\left(0, T ; L_{1+\sigma / 2}(\Omega)\right)}}{m}\right)^{1+\sigma / 2}
$$

uniformly in time. Notice that by Hölders inequality

$$
\left\|\alpha_{r}\right\|_{L_{1+\sigma / 4}(\Omega)}=\left(\int_{\text {supp } \alpha_{r}}\left|\alpha_{r}\right|^{1+\sigma / 4} d x\right)^{\frac{1}{1+\sigma / 4}} \leq\left(\left|\operatorname{supp} \alpha_{r}\right|^{\frac{\sigma}{4+2 \sigma}} \cdot\left\|\alpha_{r}\right\|_{L_{1+\sigma / 2}(\Omega)}^{\frac{4+\sigma}{4+2 \sigma}}\right)^{\frac{1}{1+\sigma / 4}} .
$$

Inequalities (2.16) and (2.15) imply

$$
\left\|\alpha_{r}\right\|_{L_{1+\sigma / 4}(\Omega)} \leq m^{-\frac{\sigma}{4+\sigma}} \cdot\left\|\alpha_{r}\right\|_{L_{1+\sigma / 2}(\Omega)}^{1-\frac{2 \sigma}{(\sigma+2)(\sigma+4)}}
$$

hence

$$
\left\|\alpha_{r}\right\|_{L_{\infty}\left(0, T ; L_{1+\sigma / 4}(\Omega)\right)} \leq m^{-\frac{\sigma}{4+\sigma}} \cdot\left\|\alpha_{r}\right\|_{L_{\infty}\left(0, T ; L_{1+\sigma / 2}(\Omega)\right)}^{1-\frac{2 \sigma}{(\sigma+2)(\sigma+4)}} .
$$

Choose $0<t_{1} \leq T$ small enough so that $4 \int_{0}^{t_{1}} f(s) d s<\frac{\sigma}{4+\sigma}$, then for $0 \leq t \leq t_{1}$ there is $y(t) \leq C m^{-\frac{\sigma}{8+2 \sigma}}$. Letting $m \rightarrow \infty$ we get $x(t)=0$ for $0 \leq t \leq t_{1}$ which reads $u_{1}^{E}=u_{2}^{E}$ for $0 \leq t \leq t_{1}$. We can continue this procedure starting at $t=t_{1}$ and get uniqueness for all $t \in[0, T]$.

Estimate (1.3) can also be used to give insight into the rate of convergence in the inviscid limit of the system (1.7).

Proof of Theorem 1.3. Let $\left\{u^{\nu}, p^{\nu}\right\}$ and $\left\{u^{E}, p^{E}\right\}$ be solutions to problems (1.7) and (1.5) respectively. Subtracting these equations we get

$$
\left(u^{\nu}-u^{E}\right)-\nu \Delta u^{\nu}+u^{\nu} \cdot \nabla\left(u^{\nu}-u^{E}\right)+\left(u^{\nu}-u^{E}\right) \nabla u^{E}=-\nabla\left(p^{\nu}-p^{E}\right) .
$$

Multiplying both sides by $\left(u^{\nu}-u^{E}\right)$ and integrating over $\Omega$ we obtain

$$
\frac{1}{2} \frac{d}{d t} \int_{\Omega}\left(u^{\nu}-u^{E}\right)^{2} d x+\nu \int_{\Omega}\left(u^{\nu}-u^{E}\right) \Delta u^{\nu} d x+\int_{\Omega}\left(u^{\nu}-u^{E}\right) u^{\nu} \nabla\left(u^{\nu}-u^{E}\right) d x
$$




$$
+\int_{\Omega}\left(u^{\nu}-u^{E}\right)^{2} \nabla u^{E} d x=-\int_{\Omega}\left(u^{\nu}-u^{E}\right) \nabla\left(p^{\nu}-p^{E}\right) d x .
$$

Integrating by parts, using boundary conditions and incompressibility of flow we reduce (2.20) to

$$
\frac{1}{2} \frac{d}{d t} \int_{\Omega}\left(u^{\nu}-u^{E}\right)^{2} d x+\int_{\Omega}\left(u^{\nu}-u^{E}\right)^{2} \nabla u^{E} d x-\nu \int_{\Omega} \nabla u^{\nu} \nabla\left(u^{\nu}-u^{E}\right) d x=0 .
$$

Let $\alpha=\left(u^{\nu}-u^{E}\right)^{2}, \beta=\nabla u^{E}$. Extending all functions by 0 outside $\Omega$, by Theorem 1.1 and Remark 2.1 we have

$$
\left|\int_{\Omega}\right| \alpha \beta|d x| \leq C\|\beta\|_{B M O}\|\alpha\|_{L_{1}(\Omega)}\left(1+\left|\ln \|\alpha\|_{L_{1}(\Omega)}\right|+\ln \left(1+\|\alpha\|_{L_{\infty}}(\Omega)\right)\right) .
$$

Let $\alpha=\alpha_{\nu}+\alpha_{r}$ so that $\left|\alpha_{\nu}\right|=\min \left(\frac{1}{\nu},|\alpha|\right)$. Proceeding as in the proof of Theorem 1.2 we denote $f(t)=2 C\|\beta(t)\|_{B M O(\Omega)}, g(t)=\int_{\Omega}\left|\alpha_{r} \beta\right| d x, x(t)=\left\|u^{\nu}-u^{E}\right\|_{L_{2}(\Omega)}^{2}$. From (2.22) we get the following inequality

$$
\begin{aligned}
& \dot{x} \leq f(t) x(t)\left(|\ln x(t)|+1+\ln \left(1+\frac{1}{\nu}\right)\right)+g(t)+\nu g_{0}(t)^{2}, \\
& x(0)=0 .
\end{aligned}
$$

To find a good estimate on $x(t)$ we introduce the following equation

$$
\begin{aligned}
& \dot{y}=f(t) y(t)\left(|\ln y(t)|+1+\ln \left(1+\frac{1}{\nu}\right)\right)+g(t)+\nu g_{0}(t)^{2}, \\
& y(0)=\nu
\end{aligned}
$$

for some $\nu$ sufficiently small. From the Osgood existence theorem we know that there exists a unique local solution to (2.24). The solution of (2.24) majorizes $x(t)$, i.e.:

$$
x(t) \leq y(t) \quad \text { for } \quad t \in[0, T] .
$$

From (2.24) we have by Gronwall's inequality

$$
\begin{gathered}
y(t) \leq \nu \exp \left(\int_{0}^{t} f(s)\left[|\ln y(s)|+1+\ln \left(1+\frac{1}{\nu}\right)\right] d s\right)+ \\
\int_{0}^{t}\left(g(s)+g_{0}(s)^{2} \nu\right) \exp \left(\int_{s}^{t} f(\tau)\left[|\ln y(\tau)|+1+\ln \left(1+\frac{1}{\nu}\right) d \tau\right]\right) d s \leq \\
\nu \exp \left(\left(1+\ln \left(1+\frac{1}{\nu}\right)\right) \int_{0}^{t} f(s) d s\right) \exp \left(\int_{0}^{t} f(s)|\ln y(s)| d s\right)+ \\
\exp \left(\left(1+\ln \left(1+\frac{1}{\nu}\right)\right) \int_{0}^{t} f(\tau) d \tau\right) \exp \left(\int_{0}^{t} f(s)|\ln y(s)| d s\right) \\
\times \int_{0}^{t}\left(g(s)+g_{0}(s)^{2} \nu\right) d s
\end{gathered}
$$


The condition $y(t) \geq \nu$ for sufficiently small $\nu$ gives $|\ln y(t)| \leq-\ln \nu=\ln \frac{1}{\nu}$. Also let $\nu$ be small enough so that $\frac{1}{\nu}\left(1+\frac{1}{\nu}\right) \leq \frac{2}{\nu^{2}}$, thus we can estimate the right hand-side of (2.25) (modulo a constant) by

$$
\begin{gathered}
\nu\left(\frac{2}{\nu^{2}}\right)^{\int_{0}^{t} f(s) d s}+\left(\frac{2}{\nu^{2}}\right)^{\int_{0}^{t} f(s) d s} \int_{0}^{t}\left(g(s)+\nu g_{0}(s)^{2}\right) d s= \\
=\left(\frac{2}{\nu^{2}}\right)^{\int_{0}^{t} f(s) d s}\left(\nu+\int_{0}^{t} \int_{\Omega}\left|\alpha_{r} \beta\right| d x d s+\nu \int_{0}^{t} g_{0}(s)^{2} d s d t\right) .
\end{gathered}
$$

Since $\alpha \in L_{\infty}\left(0, T ; L_{1+\sigma / 2}(\Omega)\right)$ and $\beta \in B M O(\Omega)$ hence $\beta \in L_{p}(\Omega)$ for any $p<\infty$ we have

$$
\int_{\Omega}\left|\alpha_{r} \beta\right| d x \leq\left\|\alpha_{r}\right\|_{L_{1+\sigma / 4}(\Omega)}\|\beta\|_{L_{(1+\sigma / 4)^{\prime}}(\Omega)}
$$

thus

$$
\begin{gathered}
\left(\frac{2}{\nu^{2}}\right)^{\int_{0}^{t} f(s) d s}\left(\nu+\int_{0}^{t} \int_{\Omega}\left|\alpha_{r} \beta\right| d x d s+\nu \int_{0}^{t}\left|g_{0}(s)\right|^{2} d s\right) \leq \\
\left(\frac{2}{\nu^{2}}\right)^{\int_{0}^{t} f(s) d s}\left(\nu+\left\|\alpha_{r}\right\|_{L_{\infty}\left(0, T ; L_{1+\sigma / 4}(\Omega)\right)}\|\beta\|_{L_{1}\left(0, T ; L_{(1+\sigma / 4)^{\prime}}(\Omega)\right)}+\nu\left\|g_{0}\right\|_{L_{2}(0, T)}^{2}\right) .
\end{gathered}
$$

From the Chebyshev inequality we notice that

$$
\left|\operatorname{supp} \alpha_{r}\right| \leq\left(\nu\|\alpha\|_{L_{\infty}\left(0, T ; L_{1+\sigma / 2}(\Omega)\right)}\right)^{1+\sigma / 2},
$$

uniformly in time. Notice that by Hölders inequality

$$
\left\|\alpha_{r}\right\|_{L_{1+\sigma / 4}(\Omega)}=\left(\int_{\text {supp } \alpha_{r}}\left|\alpha_{r}\right|^{1+\sigma / 4} d x\right)^{\frac{1}{1+\sigma / 4}} \leq\left(\left|\operatorname{supp} \alpha_{r}\right|^{\frac{\sigma}{4+2 \sigma}} \cdot\left\|\alpha_{r}\right\|_{L_{1+\sigma / 2}(\Omega)}^{\frac{4+\sigma}{4+2 \sigma}}\right)^{\frac{1}{1+\sigma / 4}} .
$$

Inequalities (2.29) and (2.28) imply

$$
\left\|\alpha_{r}\right\|_{L_{1+\sigma / 4}(\Omega)} \leq \nu^{\frac{\sigma}{4+\sigma}} \cdot\left\|\alpha_{r}\right\|_{L_{1+\sigma / 2}(\Omega)}^{1-\frac{2 \sigma}{(\sigma+2)(\sigma+4)}}
$$

hence

$$
\left\|\alpha_{r}\right\|_{L_{\infty}\left(0, T ; L_{1+\sigma / 4}(\Omega)\right)} \leq \nu^{\frac{\sigma}{4+\sigma}} \cdot\left\|\alpha_{r}\right\|_{L_{\infty}\left(0, T ; L_{1+\sigma / 2}(\Omega)\right)}^{1-\frac{2 \sigma}{(\sigma+2)(\sigma+4)}} .
$$

Choose $0<t_{1} \leq T$ small enough so that $4 \int_{0}^{t_{1}} f(s) d s<\frac{\sigma}{4+\sigma}$, then for $0 \leq t \leq t_{1}$ there is $y(t) \leq C \nu^{\frac{\sigma}{8+2 \sigma}}$. Consider now, $t_{1} \leq t \leq T$ and a problem analogous to (2.24) but 
with initial condition $y\left(t_{1}\right)=\nu^{\frac{\sigma}{8+2 \sigma}}$. Repeating all above estimates we pick $t_{1}<t_{2} \leq T$ such that $\sup _{t_{1} \leq t \leq t_{2}}\left\|u^{\nu}-u^{E}\right\|_{L_{2}(\Omega)} \leq C \nu^{\frac{\sigma}{16+4 \sigma}}$. Due to integrability of $f(t)$ iterating the procedure we eventually cover the whole interval $[0, T]$. This way we obtain the explicit rate of the convergence which depends mainly on the structure of integrability of function $f_{0}$. Thus we proved (1.9).

Assuming additionally condition (1.10) improves the result and gives an explicit uniform rate of convergence. The basic estimate presented above gives

$$
x(t) \leq C \nu\left(\frac{2}{\nu^{2}}\right)^{M t}
$$

with $M$ as in (1.10). Fix some $n \in \mathbb{N}$ and consider $0 \leq t \leq T /(2 M n)$. We have $\sup _{0 \leq t \leq T /(2 M n)}\left\|u^{\nu}-u^{E}\right\|_{L_{2}(\Omega)} \leq C \nu^{1-T / n}$. The time interval has been divided into $2 M n$ parts and repeating the estimate we get

$$
\sup _{0 \leq t \leq T}\left\|u^{\nu}-u^{E}\right\|_{L_{2}(\Omega)} \leq C \nu^{(1-T / n)^{2 M n}}
$$

and taking limit $n \rightarrow \infty$ we obtain

$$
\sup _{0 \leq t \leq T}\left\|u^{\nu}-u^{E}\right\|_{L_{2}(\Omega)} \leq C \nu^{e^{-2 M T}} .
$$

Theorem 1.3 is proved.

Acknowledgments. The first author (PBM) has been partly supported by Polish KBN grant No. 1 P03A 02130.

\section{References}

[1] Clopeau T., Mikelić A., Robert R., On the vanishing viscosity limit for the 2D incompressible NavierStokes equations with friction type boundary conditions. Nonlinearity 11 (1998), 1625-1636.

[2] Constantin P., Wu J., Inviscid limit for vortex patches. Nonlinearity 8 (1995), 735-742.

[3] Fefferman C., Stein E.M., $H^{p}$ spaces of several variables. Acta Math. 129 no. 3-4 (1972), 137-193.

[4] Hartman F., Ordinary differential equations. John Wiley \& Sons, NY-London-Sydney (1964).

[5] Kato T., On classical solutions of the two-dimensional nonstationary Euler equation. Arch. Rational Mech. Anal. 25 (1967), 188-200.

[6] Kozono H., Taniuchi Y., Limiting case of the Sobolev inequality in BMO, with application to the Euler equations. Comm. Math. Phys. 214 no. 1 (2000), 191-200.

[7] Mucha P.B.,On the inviscid limit of the Navier-Stokes equations for flows with large flux. Nonlinearity 16 no. 5 (2003) 1715-1732. 
[8] Rusin W.M., On the inviscid limit for the solutions of two-dimensional incompressible Navier-Stokes equations with slip-type boundary conditions. Nonlinearity 19 no. 6 (2006), 1349-1363.

[9] Torchinsky A., Real-variable methods in harmonic analysis. Pure and Applied Mathematics, 123. Academic Press, Inc., Orlando, FL, 1986.

[10] Yudovich V., Nonstationary flow of an ideal incompressible liquid. Zhurn. Vych. Mat. 3 (1963), $1032-1066$.

[11] Yudovich V., Uniqueness theorem for the basic nonstationary problem in the dynamics of an ideal incompressible fluid. Math. Res. Lett. 2 (1995), 27-38.

[12] Vishik M., Incompressible flows of an ideal fluid with vorticity in borderline spaces of Besov type. Ann. Sci. cole Norm. Sup. (4) 32 no. 6 (1999), 769-812.

[13] Zygmund A., Trygonometric Series. Cambridge Univ. Press, London-NY, 1959. 